\title{
Riqueza y distribución vertical de los moluscos del litoral rocoso de la provincia de Islay, Arequipa, al sur del Perú
}

\section{Mollusk richness and vertical distribution along the rocky shore of Islay, Arequipa, Southern Peru}

\author{
Cristian A. Tejada-Pérez ${ }^{1 *}$, Francisco Villasante ${ }^{2,3}$, Cesar Luque-Fernández ${ }^{2,3}$, \\ Claudia L. Tejada-Begazo ${ }^{2,4}$ y Michael Espinoza ${ }^{2}$
}

\begin{abstract}
RESUMEN
El litoral rocoso es un ecosistema heterogéneo en el que se distinguen una franja supralitoral, una zona mediolitoral y una franja infralitoral, al reconocer a las especies de moluscos como indicadoras de una zonación vertical. Este trabajo contribuye al conocimiento de la riqueza y la distribución vertical de los moluscos intermareales para la provincia de Islay, Arequipa, Perú. Se realizó una búsqueda intensiva en los meses de noviembre y diciembre del año 2013, para poder determinar la riqueza y se establecieron tres cuadrantes de 25 x $25 \mathrm{~cm}$ de lado distribuidos perpendicularmente a la línea costera con 14 repeticiones en los 7 sitios de estudio, para establecer su distribución vertical. Se utilizó un análisis de componentes principales (ACP), un escalamiento multidimensional no métrico (nMDS) y curvas de rango-abundancia para la determinación de la distribución vertical, y un análisis de similaridades (ANOSIM) para determinar diferencias entre la composición de moluscos entre los sitios de estudios y las subzonas del litoral. Se encontraron un total de 57 taxones, donde Echinolittorina peruviana fue la especie más representativa en la franja supralitoral, Perumytilus purpuratus para la zona mediolitoral y Tegula atra para la franja infralitoral. No se presentaron diferencias significativas en la composición de moluscos entre los sitios de estudio, pero sí entre las subzonas, al ser la zona mediolitoral la que presentó mayor riqueza y abundancia de organismos. La similitud fue mayor entre las subzonas medio e infra- con respecto a la supralitoral.
\end{abstract}

Palabras claves: Intermareal, Matarani, zonación, Echinolittorina, Catarindo.

1 Instituto Científico Michael Owen Dillon-IMOD, Calle Los Arces 339, Cayma, Arequipa, Perú ctejada@imod.org.pe*

2 Departamento Académico de Biología, Universidad Nacional de San Agustín de Arequipa, Av. Alcides Carrión s/n, Arequipa, Perú.

3 Instituto de Ciencia y Gestión Ambiental-Universidad Nacional de San Agustín de Arequipa (ICIGA-UNSA), Calle San Agustín 108, Arequipa, Perú.

4 Laboratorio de Ciencias del Mar N. ${ }^{3}$ 317, Universidad Peruana Cayetano Heredia, Av. Honorio Delgado 430, San Martín de Porres, Lima, Perú.

Recibido: 2 octubre 2017

Corregido: 8 marzo 2018

Aceptado: 13 marzo 2018

DOI: http://dx.doi.org/10.15359/revmar.10-1.4

Rev. Mar. Cost. ESSN 1659-407X. Vol. 10 (1): 47-66, Enero-Junio 2018. 


\section{ABSTRACT}

The coastline is a heterogeneous ecosystem that includes a supralittoral fringe, a midlittoral zone, and an infralittoral fringe, with mollusk species that serve as indicators of vertical zonation. This study contributes to the knowledge of richness and vertical distribution of intertidal mollusks in Islay, Arequipa, Peru. An intensive search was conducted during November and December 2013 to determine richness. A total of $325 \times 25 \mathrm{~cm}$ grids where located perpendicularly to the seashore with 14 repetitions in each of the 7 study sites, to establish their vertical distribution. A Principal Component Analysis (PCA), nonmetric multidimensional scaling (nMDS) and rank-abundance curves were used to determine vertical distribution. In addition, an Analysis of Similarities (ANOSIM) was utilized to determine differences between mollusk compositions between study sites and subzones. A total of 57 taxa were found, where Echinolittorina peruviana was the most representative species in the supralittoral fringe, Perumytilus purpuratus in the midlittoral zone and Tegula atra in the infralittoral fringe. No significant differences were found in the mollusk composition between study sites. However, there were differences among sub-areas, where the midlittoral zone had the greatest richness and abundance of organisms. There were more similarities between the middle and infra-subzones with respect to the supralittoral fringe.

Keywords: Intertidal, Matarani, zonation, Echinolittorina, Catarindo.

\section{INTRODUCCIÓN}

Según Underwood (1981), la línea costera rocosa intermareal es un ambiente muy heterogéneo que soporta una amplia variedad de formas de vida las cuales se distribuyen de forma particular, al seguir un patrón de zonación característico verticalmente (Stephenson \& Stephenson, 1949). De todos los grupos de organismos que habitan el litoral rocoso, los moluscos han sido reconocidos como indicadores de zonación específica (Bandel \& Wedler, 1987). Existen factores físicos y biológicos que modifican de diferente forma esta zonación, al ser en la franja supralitoral, los factores físicos como la desecación y la radiación solar son los que limitan el número de especies, y los factores biológicos como la depredación y la competencia de espacio son los que modifican la franja infralitoral. La zona mediolitoral es una combinación de ambas (Castro \& Huber, 2003).

Para Álamo \& Valdivieso (1997), el Perú es un país que posee una de las más altas concentraciones de diversidad biológica debido a su complejidad ecológica y biogeográfica; sin embargo, la mayoría de estudios sobre phyllum Mollusca se han realizado en Provincia Panameña o Provincia del Pacífico Oriental Tropical (Spalding et al. 2007). Por esta razón, en la zona norte de Perú se reporta la mayor riqueza del moluscos (Ramírez et al. 2003); por otro lado, Paredes et al. (1999), menciona que la principal parte de información generada sobre ellos corresponde al departa- 
mento de Lima e Ica que pertenecen a la Provincia Peruana o Provincia del Pacífico Sudeste Templado-Cálido (Spalding et al. 2007). Para el departamento de Arequipa, ubicado en la zona sur del Perú, existe solamente un estudio referido a moluscos, realizado en la provincia de Islay por Talledo y Gonzales (1989).

La distribución vertical de los invertebrados marinos en la zona costera de Perú son aún más escasos, y solo se cuenta con el de Paredes (1974), para el departamento de Lima, en donde se propone un esquema zonacional modificado que se basa en Stephenson y Stephenson (1949).

Debido a la escasa información, esta investigación contribuye al conocimiento de la diversidad de moluscos y su distribución vertical largo de la costa rocosa de la provincia de Islay-Arequipa.

\section{MATERIAL Y MÉTODOS}

\section{Área de estudio}

La provincia de Islay abarca una sección de la línea costera del departamento de Arequipa, al sur del occidente de Perú, y posee un litoral que corre en dirección noroeste-sureste. Desde el límite con la provincia de Camaná hasta Mollendo, se presentan acantilados bajos, "caletas" pequeñas, islotes y pequeñas playas arenosas o pedregosas, que luego continúa en un litoral arenoso desde Mollendo hasta Corío, interrumpido por la desembocadura del río Tambo y algunas pequeñas formaciones rocosas en Mejía, para luego continuar con un litoral rocoso desde Corío hasta el departamento de Moquegua (Hughe, 1991).

Se estudiaron 7 sitios a lo largo del litoral rocoso de la provincia de Islay, los cuales correspondieron a caletas (Fig. 1). Estas poseen pequeñas playas de arena o canto rodado, comprendido de dos acantilados no muy pronunciados. Todos los sitios de estudio presentan condiciones físicas similares, como una zona costera rocosa semiprotegida y muy poco intervenida por acción antrópica. El muestreo se enfocó en superficies de $50^{\circ}-80^{\circ}$ grados de inclinación, sobre sustrato rocoso, de superficie lisa

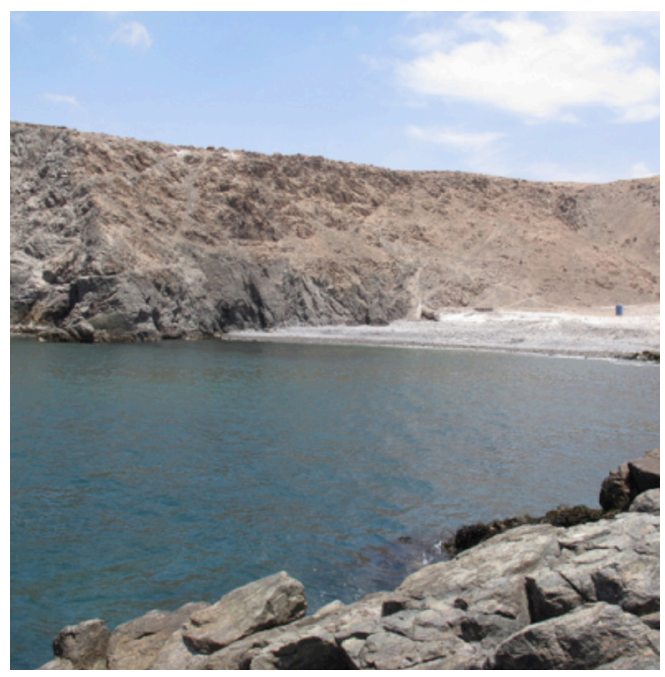

Fig. 1. Fotografía de la caleta "Playa Mollendito" en Islay, Arequipa- Perú Fig. 1. Photograph of the cove at "Playa Mollendito", Islay, Arequipa-Peru 
o rugosa y con o sin cobertura algal. Los 7 sitios de estudio fueron: Mollendito (MOL) (16 58' 09' S y $72^{\circ}$ 07'09” O), Punta Islay-El Faro (PUN) (17 00' 51" S y 72 06' 32" O), Dos Playas (DOS) $\left(17^{\circ} 00^{\prime} 53^{\prime \prime} \mathrm{S}\right.$ y $72^{\circ}$ 04' 52" O), La Ballenita (BAL) (17 00' $54^{\prime \prime}$ S y 72 $02^{\circ}$ ' 30" O), Catarindo

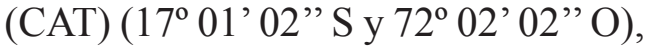
Calera (CAL) $\left(17^{\circ} 15^{\prime} 13^{\prime \prime}\right.$ S y $72^{\circ} 32^{\prime}$ 33 " O) y Pacae (PAC) $\left(17^{\circ} 15\right.$ ' 53" S y $72^{\circ} 31^{\prime} 26^{\prime \prime} \mathrm{O}$ ) (Fig. 2) y se realizaron muestreos durante los meses de noviembre y diciembre del 2013.

\section{Metodología}

La riqueza de moluscos se determinó a través de una búsqueda intensiva en cada sitio de estudios, en todos los tipos de ambientes en el litoral durante pleamar y bajamar (Guzmán et al. 1998).

Para la distribución vertical, se utilizó tres cuadrículas de $25 \times 25 \mathrm{~cm}$ de lado $\left(625 \mathrm{~cm}^{2}\right)$ distribuidas verticalmente en el litoral al seguir la metodología propuesta por Jones (1980), quien toma como límite superior a los litorínidos y como límite inferior a los equinodermos,

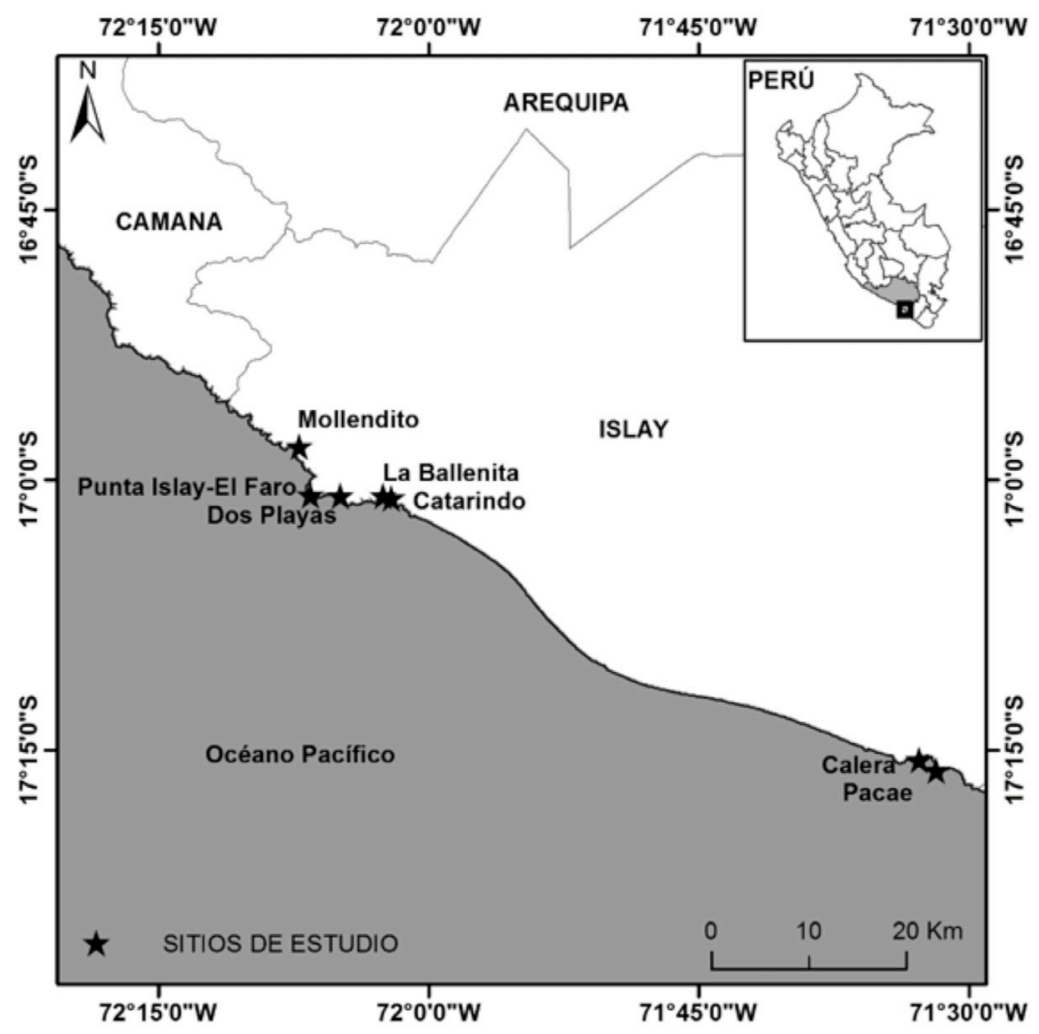

Fig. 2. Mapa de los sitios de estudio en la provincia de Islay, Arequipa-Perú Fig. 2. Map of study sites in the province of Islay, Arequipa-Peru 
y también se tomó como referencia el esquema de Paredes (1974) (Fig. 3). Se contabilizarón todos los moluscos dentro de cada cuadrícula, las cuales se colocaron: uno en la franja supralitoral, otra en la zona mediolitoral y otra en la franja infralitoral (Osorio \& Cantuarias, 1989), a las que se denominaron como "subzona", para este trabajo (Sibaja-Cordero, 2008; Sibaja-Cordero \& Cortés, 2008; Londoño-Cruz et al. 2014; Ojeda et al. 2014). El tamaño de cada subzona fue muy variable, por lo que se tomó un punto medio referencial por cada una de ellas, para no tener preferencias de ningún tipo al momento de colocar la cuadricula. Se realizaron 14 repeticiones (las 3 cuadrantes verticalmente) distanciadas entre 10 a 15 metros en cada una de los 7 sitios de estudio.

Tanto en la búsqueda intensiva y en los cuadrantes, los organismos que no pudieron ser determinados en campo fueron trasladados al laboratorio para su determinación a posteriori. Para la determinación taxonómica se utilizó a Marincovich (1973), McLean (1984), Paredes (1980; 1986), Talledo y Gonzales (1989), Álamo y Valdivieso (1997), Guzmán

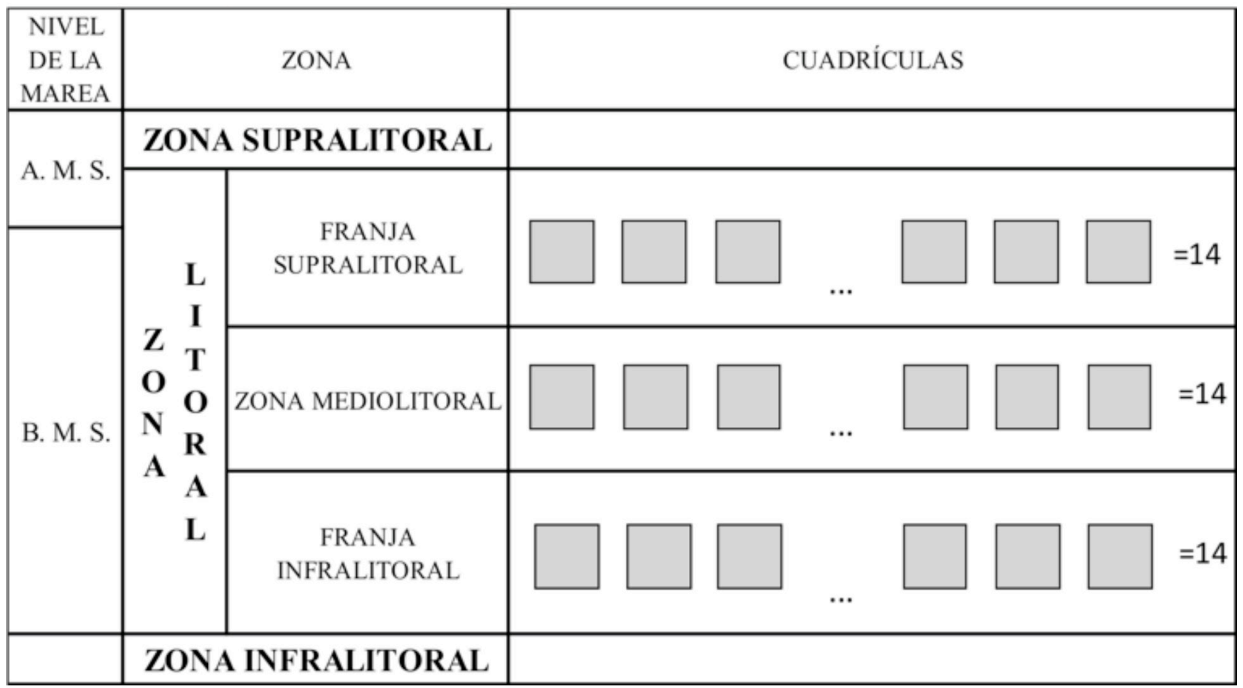

Fig. 3. Esquema propuesto por Paredes (1974), de la zona litoral y la ubicación de las cuadrículas perpendicularmente al mar en cada sitio de estudio. A. M. S.: Pleamar de sicigias, B. M. S.: Bajamar de Sicigias

Fig. 3. Scheme proposed by Paredes (1974), for the rocky seashore and location of the grids for each study site. A.M.S.: high tide of Syzygy, B.M.S.: Syzygy's low tide 
et al. (1998), Veliz y Vásquez (2000), Espoz et al. (2004), Aldea y Valdovinos (2005), Veliz et al. (2012), Güller y Zelaya (2013), y a Schrödl y Hooker (2014). El arreglo taxonómico fue con base en World Register of Marine Species (WORMS Editorial Board, 2017) y Worldwide Mollusc Species Data Base (WMSDB) (Aldea et al. 2011; Galli 2013).

En el análisis de datos, la riqueza fue representada con el número total de taxones encontrados por sitio de estudio y en total para la provincia de Islay (Krebs, 1985; Torreblanca-Ramírez et al. 2012). Se aplicó un análisis de componentes principales (ACP) al transformar la abundancia en Log $(\mathrm{X}+1)$ (Sibaja-Cordero, 2008; Sibaja-Cordero \& Cortés, 2008), para analizar la distribución vertical de las especies. Además, se elaboraron curvas de rango-abundancia $(\log 10(\mathrm{X}+1))$ (Sokal \& Rohlf, 1981), para cada subzona del litoral rocoso (Feinsinger, 2004). Se empleó un escalamiento multidimensional no métrico (nMDS) con la abundancia transformada a la doble raíz cuadrada (Clarke \& Warwick, 2001), con la distancia de disimilaridad de Bray-Curtis (Clarke \& Warwick, 2001; Sibaja-Cordero 2008; Sibaja-Cordero \& Cortés, 2008), para establecer las similitudes y/o la disimilitudes entre las 3 subzonas: la franja supralitoral, la zona mediolitoral y la franja infralitoral, de los sitios estudiados con respecto a la abundancia de las especies y se complementó con una prueba de análisis de similitudes (ANOSIM) (Clarke \& Warwick, 2001), para determinar si la composición de moluscos en los cuadrantes fueron similares entre sitios $(\mathrm{R}>0, P$ $<0.01)$. El valor de $\mathrm{R}$ determina el nivel de diferencia donde $\mathrm{R}=1$ indica que existe una total diferencia entre grupos. Para el análisis estadístico se utilizó el programa libre PAST versión 2.17c (Hammer et al. 2001).

\section{RESULTADOS}

\section{Diversidad}

Se contabilizó un total de 17740 especímenes de moluscos dentro de los cuadrantes de muestreo, sin considerar a los organismos que se encontraron en la búsqueda intensiva. Todos los moluscos se agrupan en 4 clases, 29 familias, 42 géneros y 57 taxones (Cuadro 1). La clase Gastropoda fue la mejor representada con 15 familias y 31 taxones (54.39\%), seguida de Bivalvia con 10 familias y 17 taxones (29.82\%). La clase Polyplacophora comprendió únicamente 3 familias y 8 taxones $(14.04 \%)$ y la clase Cephalopoda con una familia y un taxón $(1.75 \%)$. Las familias mejores representadas son Lottiidae (7 taxones), Chitonidae (6 taxones) y Fissurellidae (6 taxones) (Fig. 4). El sitio de estudio Dos Playas posee la mayor riqueza con 49 taxones y la menor es Pacae con 26 taxones (Fig. 5). 


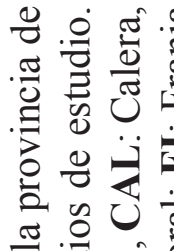

ฮี

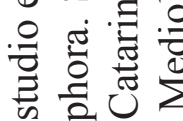

0

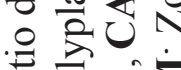

के

焉

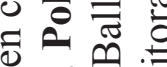

论

号 की

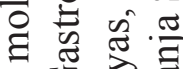

ष

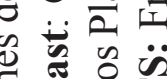

Оี

芯

¿ 융

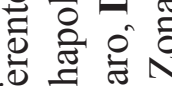

प्ते

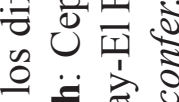

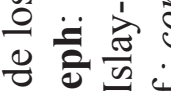

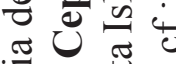

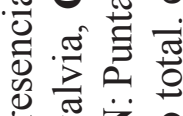

穷.

的

势

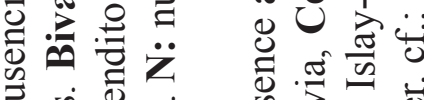

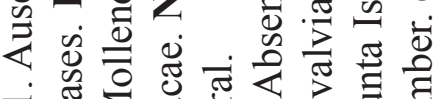

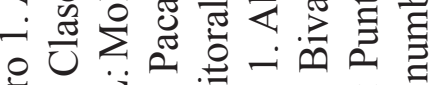

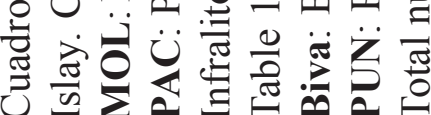

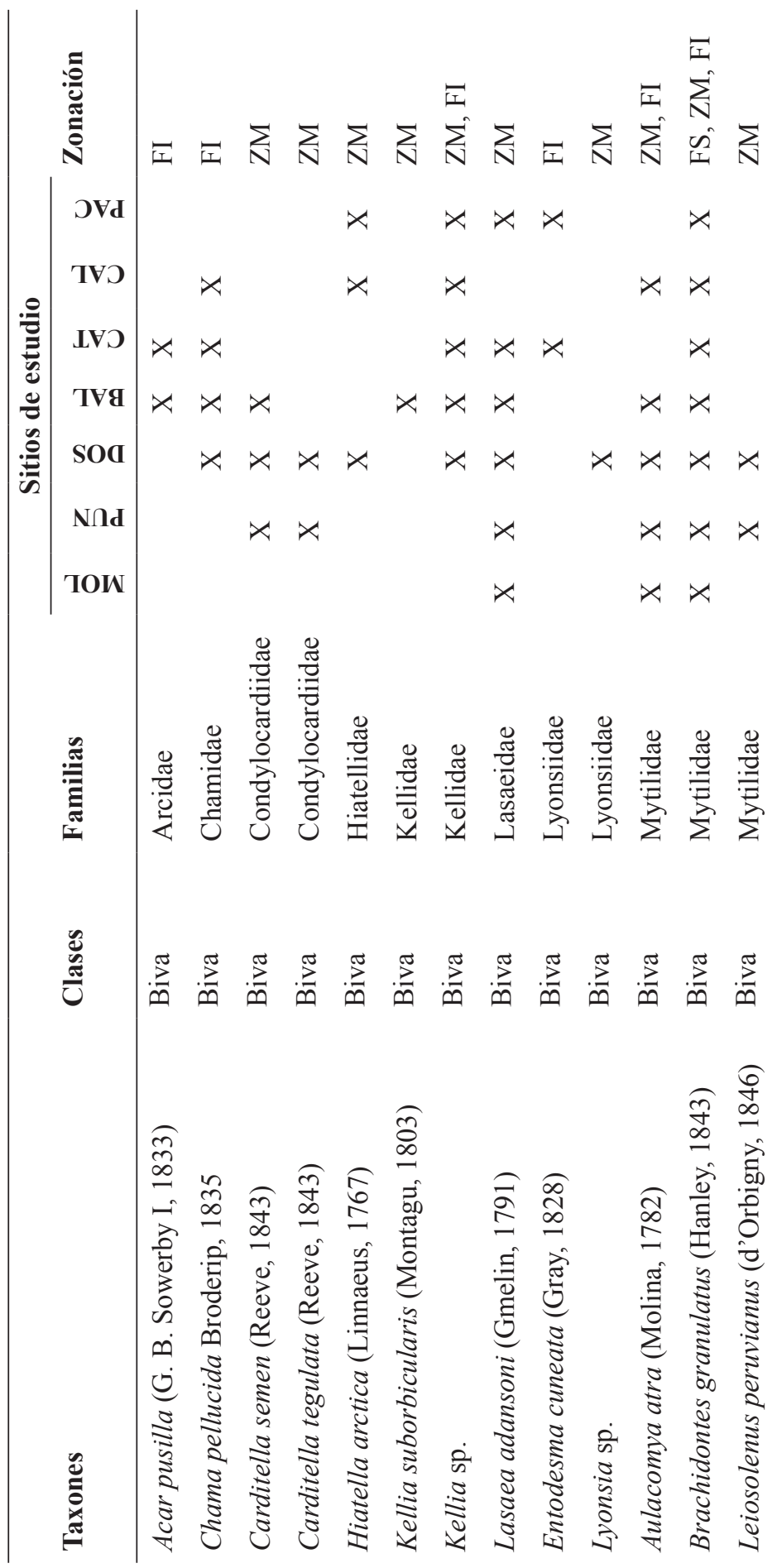




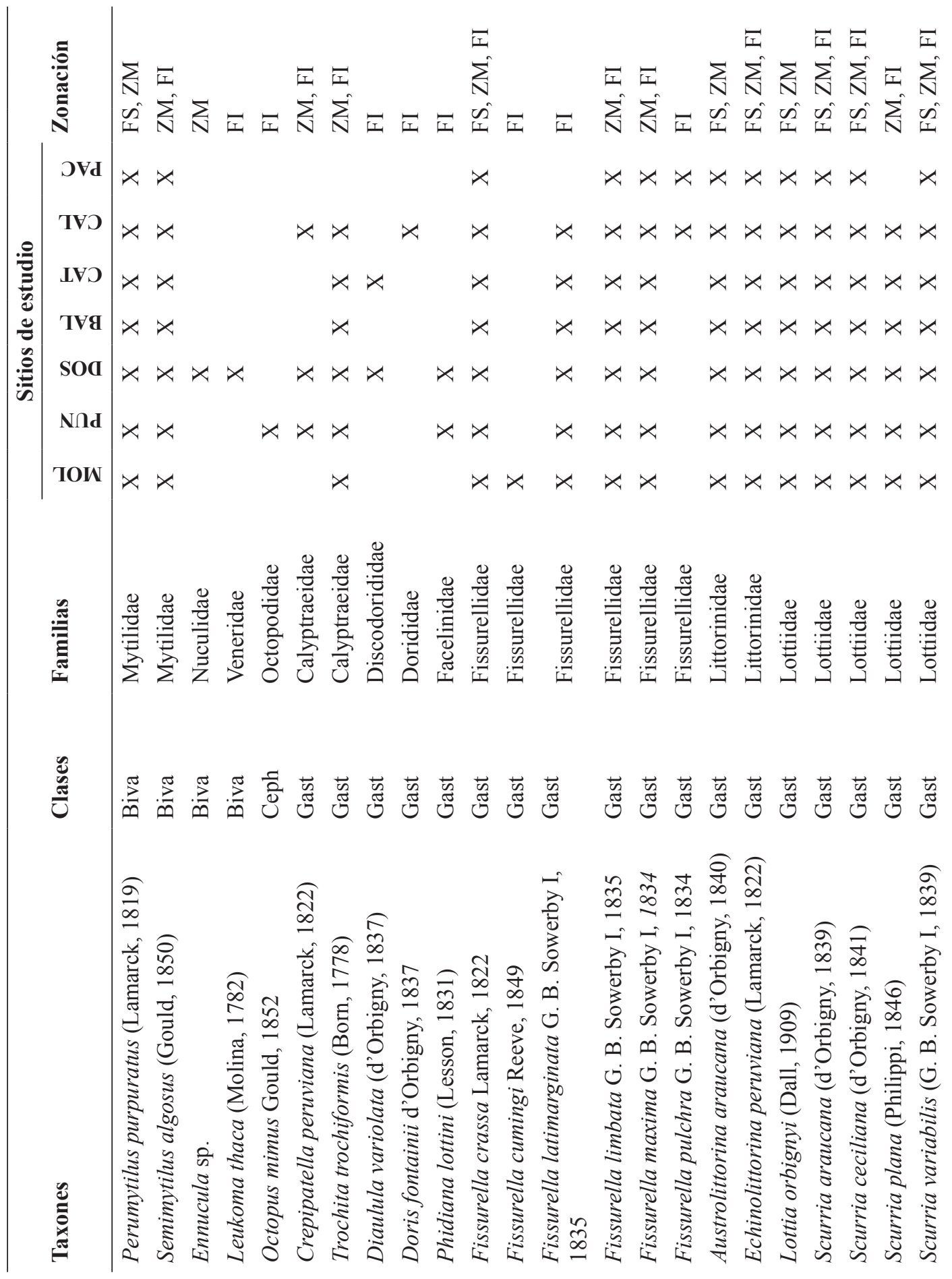




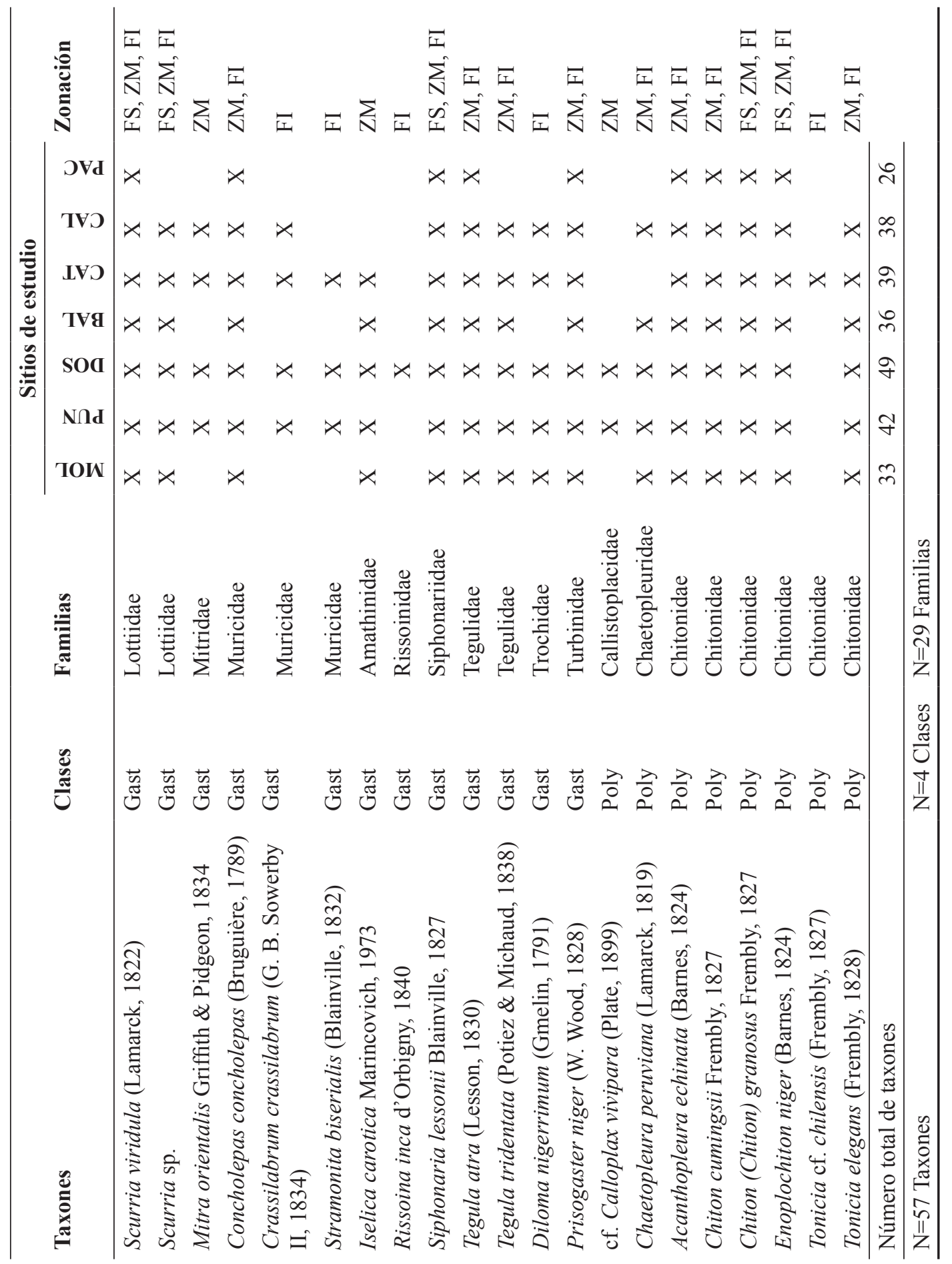




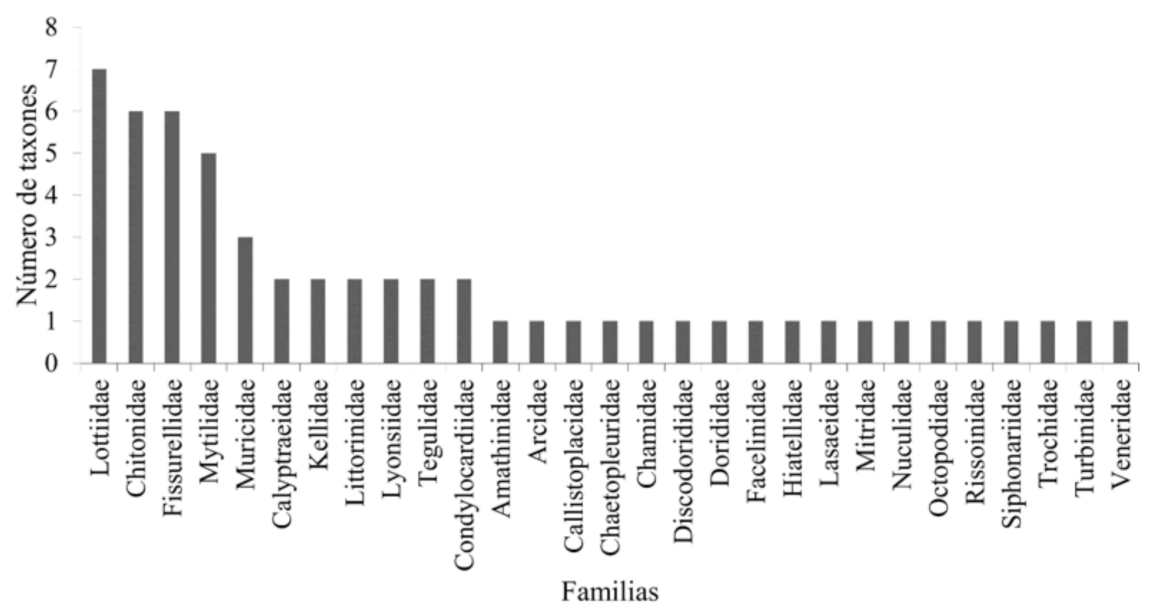

Fig. 4. Familias de moluscos ordenados de acuerdo al número de taxones para la provincia de Islay, Arequipa

Fig. 4. Mollusk families ordered according to the number of taxa for the Islay province, Arequipa

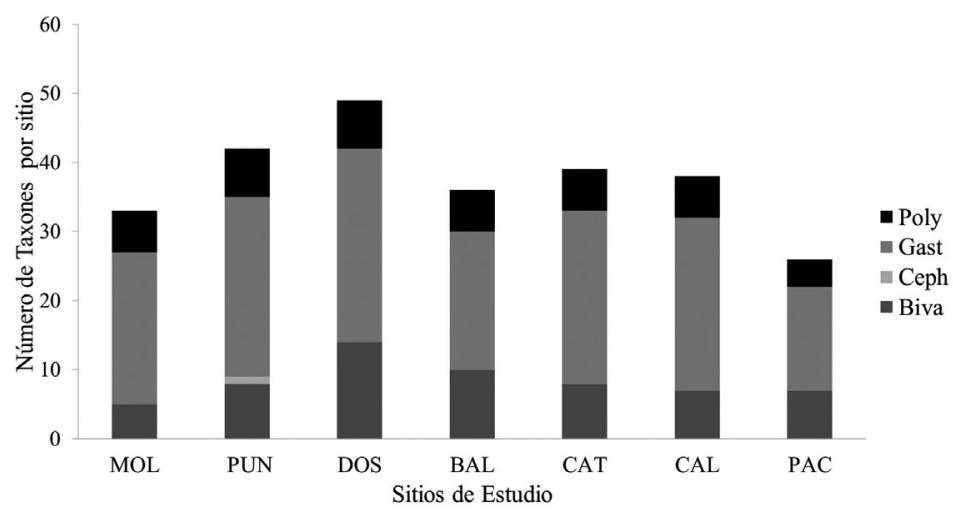

Fig. 5. Números de taxones y clases en cada sitio de estudio en la provincia de Islay. MOL: Mollendito; PUN: Punta Islay-El Faro; DOS: Dos playas; BAL: Ballenitas; CAT: Catarindo; CAL: Calera; PAC: Pacae. Clases: Poly: Polyplacophora; Gast: Gastropoda; Ceph: Cephalopoda; Biva: Bivalvia Fig. 5. Number of taxa and classes in each study site for the province of Islay. MOL: Mollendito; PUN: Punta Islay-El Faro; DOS: Dos playas; BAL: Ballenitas; CAT: Catarindo; CAL: Calera; PAC: Pacae. Clases: Poly: Polyplacophora; Gast: Gastropoda; Ceph: Cephalopoda; Biva: Bivalvia 


\section{Distribución vertical}

El análisis de escalamiento multidimensional no paramétrico (nMDS) de dos dimensiones $(R=0.3058)$ muestra la existencia de una disimilaridad entre las tres subzonas del litoral, sobre todo de la franja supralitoral con respecto a las otros dos restantes (Fig. 6).

El análisis de componentes principales (ACP) reveló que ciertas especies están más representadas en cada una de las subzonas del litoral rocoso. El porcentaje total de varianza acumulada de los dos primeros componentes fue $59.62 \% \quad(\mathrm{PCl}$ :
$38.57 \%$ y PC2: $21.053 \%$ ). Para la franja supralitoral E. peruviana (EchiPeru) es la especie que se encuentra más representada para esta subzona, seguida de $A$. araucana (AustArau). Para la zona mediolitoral se encuentra $P$. purpuratus (PeruPurp) con $S$. algosus (SemiAlgo). Finalmente, para la franja infralitoral T. atra (TeguAtr) es la mejor representada seguida por P. niger (PrisNige). Por otro lado, hay ciertas especies que se comparten entre la zona mediolitoral y la franja infralitoral, al ser esta principalmente T. tridentata (TeguTrid) (Fig. 7).

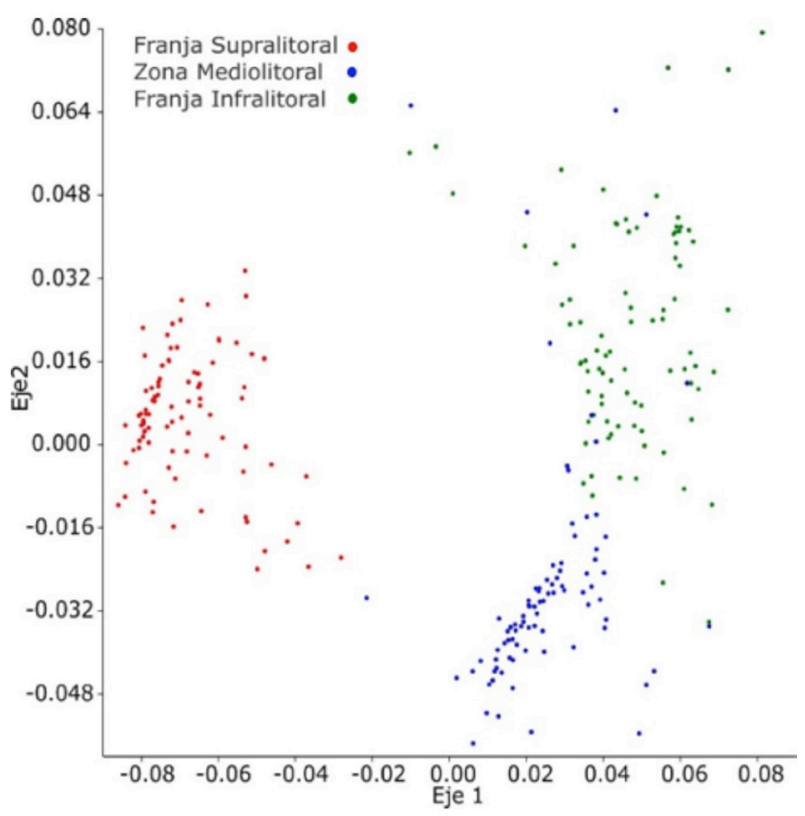

Fig. 6. Escalamiento multidimensional no métrico (nMDS), para la zonación vertical de los moluscos del litoral rocoso en Islay, Arequipa

Fig. 6. Non-metric multidimensional scaling (nMDS) for vertical zonation of mollusks along the rocky shore in Islay, Arequipa 


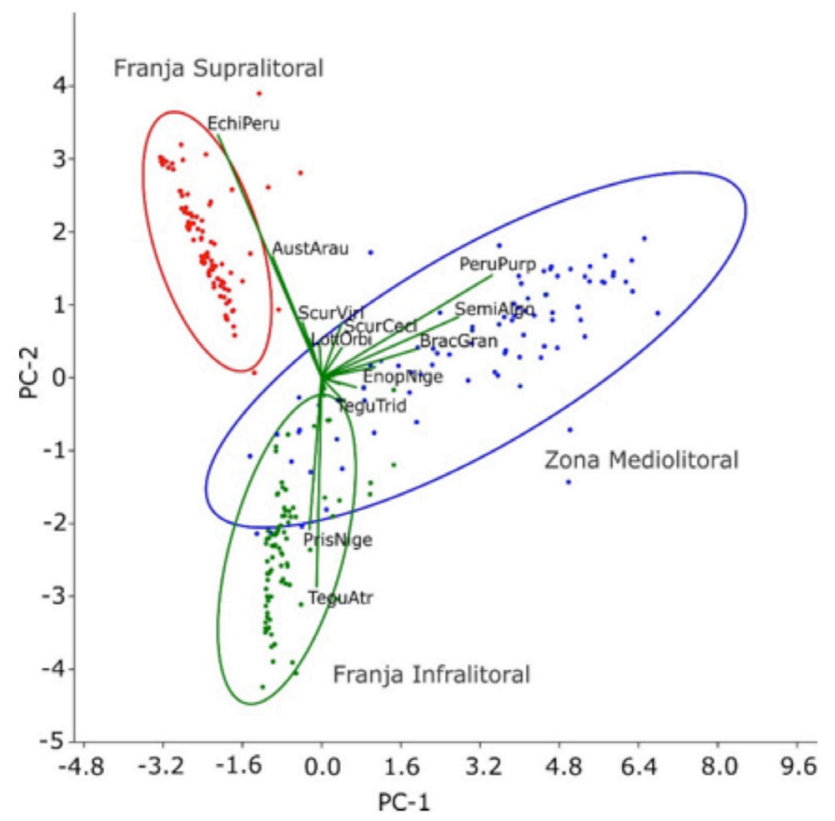

Fig. 7. Análisis de Componentes Principales (ACP), para la zonación vertical de los moluscos del litoral rocoso en Islay, Arequipa. Se señalan las subzonas identificadas (círculos) y la variación de la abundancia en cada una de estas (vectores)

Fig. 7. Principal Component Analysis (PCA) for vertical zonation of mollusks along the rocky shore in Islay, Arequipa. The identified subareas (circles)and abundance variations are marked in each (vectors)

La diversidad específica encontrada en cada subzona del litoral es diferente entre sí, ya que la mayor riqueza y diversidad se concentra en la zona mediolitoral seguida de la franja infralitoral y la supralitoral respectivamente. En las tres subzonas existe dominancia de ciertas especies, tal es el caso de $E$. Peruviana (520.36 ind. $\mathrm{m}^{-2}$ ) y A. araucana (145.78 ind. $\mathrm{m}^{-2}$ ) que presentan la mayor abundancia de organismos en la franja supralitoral. Para la zona mediolitoral P. purpuratus (958.12 ind. $\mathrm{m}^{-2}$ ), S. algosus (459.48 ind. $\mathrm{m}^{-2}$ ) y $B$. granulatus (210.64 ind. $\mathrm{m}^{-2}$ ) se presentaron como las especies abundantes. Finalmente, la franja infralitoral está dominada por T. atra (187.59 ind. $\left.\mathrm{m}^{-2}\right)$ y P. niger (152.83 ind. $\left.\mathrm{m}^{-2}\right)$.

Sin embargo, a pesar de existir especies dominantes, las curvas de rango-abundancia muestran una mayor equidad (Fig. 8) con una menor pendiente entre la zona mediolitoral y la franja infralitoral, con respecto a la franja supralitoral. 


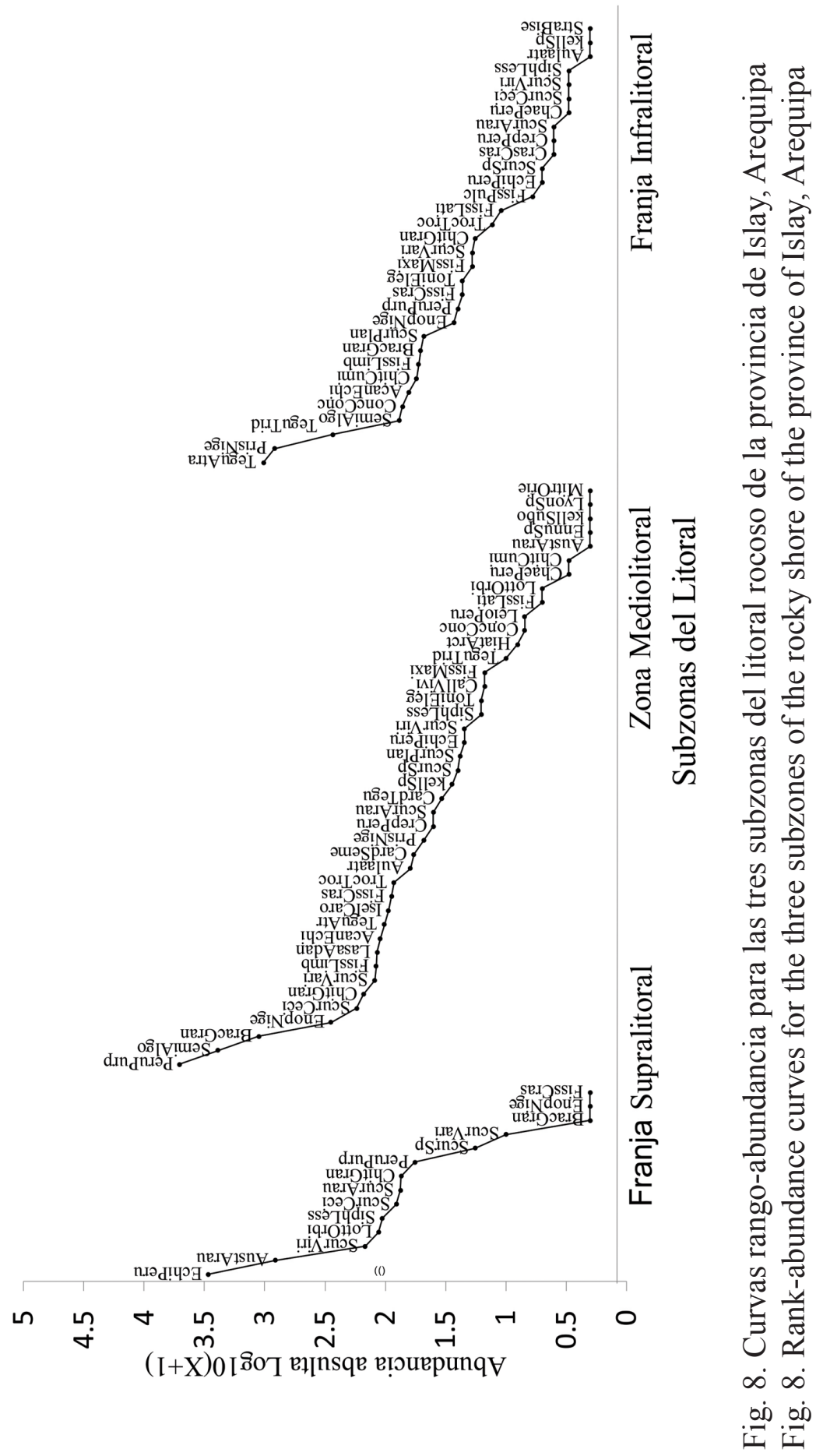


El análisis de similitud (ANOSIM) muestra que no existe una diferencia significativa $(R=0.006, P=$ 0.217 ) en la composición de moluscos litorales entre las sitios de estudio, mientras que entre las subzonas del litoral si presenta una diferencia significativa $(R=0.86, P=0.001)$. Entre las subzonas del litoral, la mayor diferencia es entre la franja supralitoral con la franja infralitoral $(R=0.9415, P=$ 0.001 ) y la menor diferencia es entre la zona mediolitoral con la franja infralitoral $(R=0.6992, P=0.001)$.

\section{DISCUSIÓN}

Del total de los 57 taxones de moluscos encontrados para la zona de estudio, algunos resultan ser nuevos registros para el área como: $C h$. peruviana, $D$. variolata, $D$. fontainii, $P$. lotinii, F. cumingi, F. pulchra, S. plana, I. carotica, $R$. inca, cf. C. vivipara, $T$. cf. chilensis y $T$. elegans; ya que Talledo y Gonzales (1989), no los registraron en su estudio. Paredes et al. (1999), reporta 79 taxones de moluscos para el departamento de Lima, el cual se ubica en la misma provincia biogeográfica, y la diferencia se debería al mayor tiempo de muestreo que realizaron de 1970 a 1974; ya que el tiempo y espacio es un factor importante en un estudio (Fernández \& Jiménez, 2006). La estacionalidad parece no afectar notoriamente la abundancia y la rique- za de los moluscos según Ojeda et al. (2014), ya que en su estudio los móviles tienden a aumentar ligeramente su densidad durante el verano austral y en los filtradores sésiles se mantienen casi constante $\mathrm{y} / \mathrm{o}$ incluso registran su máxima abundancia en invierno. Otro factor importante por considerar para un inventario taxonómico es la sobreevaluación de macromoluscos (especies conspicuas), donde los micromoluscos (taxones menores de 10 $\mathrm{mm}$ ) suelen ser ignorados debido a la dificultad que representa su determinación taxonómica (Esqueda-González et al. 2014).

Los patelogastrópodos Scurria zebrina y Scurria scurra registrados para Perú por Álamo y Valdivieso (1997), Paredes (1980) y Talledo y Gonzales (1989), no se encontraron en la provincia de Islay, al coincidir con lo reportado por Espoz et al. (2004). S. zebrina presenta una morfología externa similar a los juveniles de $S$. viridula lo que conlleva muchas veces a una mala determinación y $S$. scurra habita en el infralitoral. Además, ambas especies están restringidas para costas chilenas (Espoz, 2002; Espoz et al. 2004).

$S$. variabilis fue encontrada en sus diferentes ecofenotipos como lo menciona Espoz et al. (2004), donde afirma que Scurria parasitica resulta ser uno de estos, aunque Aldea y Valdovinos (2005), sugieren aún que debieran ser consideradas las dos como especies diferentes por las caracterís- 
ticas externas de la concha. Del mismo modo, se tiene a $S$. ceciliana, y a su forma infectada por el liquen Thelidium litorale, que erróneamente era considerada como Scurria bohemita debido al liquen que le otorga un aspecto similar a los cirrípedos intermareales (Espoz et al. 1995). Espoz et al. (2004), mencionan que $S$. bohemita corresponde en realidad a $S$. ceciliana.

Se registró a $C$. peruviana, confundida antes como Crepipatella dilatata, especie comúnmente citada en la literatura peruana por su alta similitud en las características externas de la concha, pero esta última únicamente se encuentra distribuida por el sur de Chile y Argentina (Veliz et al. 2012). También, el registro de $C$. tegulata coincide con Dall (1910), Paredes et al. (2016) y Ramírez et al. (2003), como una especie que habita la costa peruana, aunque según Güller y Zelaya (2013), mencionan que no se encuentra registrada para Perú y que su límite norte está en Valparaíso $\left(33^{\circ} \mathrm{S}\right)$ Chile. Por lo tanto, este trabajo confirma el registro de $C$. peruviana y $C$. tegulata para las costas de Perú.

Con relación en la distribución vertical de los moluscos en el litoral rocoso, es evidente que algunas especies son representativas para la franja supralitoral, otras para la zona mediolitoral y otras para la franja infralitoral, y ello debido a los factores físicos y biológicos a los que están sometidos (Castro \& Huber, 2003). La abundan- cia y la riqueza específica entre las tres subzonas del litoral en el presente estudio presentaron diferencias significativas de acuerdo a los resultados de la prueba de ANOSIM. Chappuis et al. (2014), mencionan que la variación vertical es más marcada que si esta fuese horizontal dentro de una misma área biogeográfica, en el que un estudio es llevado y ello debido a que entre los diferentes sitios de estudio comparten factores físicos similares, como temperatura del agua y salinidad. En el caso de la franja supralitoral E. peruviana y $A$. araucana son las especies más representativas descritas también para la costa central del Perú por Paredes (1974). De acuerdo a Vegas-Vélez (1971) y Osorio y Cantuarias (1989), las especies de litorínidos están especialmente adaptadas a soportar altas temperaturas y al estrés por desecación, debido a que estos organismos tienen la habilidad de conservar agua en sus cuerpos para disipar el calor (Wieser, 1980; Shick et al. 1988); Ahmad et al. (2011), mencionan que algunas especies de litorínidos producen mucus para reducir el contacto con la roca caliente y al mismo tiempo se deslizan dentro de su concha en bajamar. Estudios similares también describen a litorínidos como las especies predominantes en esta subzona del litoral (ej.: Chelazzi \& Vannini, 1980; Osorio \& Cantuarias, 1989; Esqueda et al. 2000; Jiménez et al. 2004; Sibaja-cordero \& Cortés, 2008). Incluso Stephenson y 
Stephenson (1949), reconocieron un patrón general en esta subzona y la denominaron como la "Zona Litorina", y Moore (1958), señala que la distribución de estos organismos es a escala mundial. McQuaid (1996), señala que estas especies se adaptaron a una variedad de hábitats al soportar distintas condiciones en términos de tolerancia térmica, lo que permite tomarlos como referencia en los esquemas de zonación vertical con relación en la temperatura, la desecación y el oleaje. Por otro lado, $P$. purpuratus es la especie más representativas para la zona mediolitoral, seguida de $S$. algosus y $B$. granulatus, estos resultados fueron encontrados similares con el litoral del centro del Perú (Paredes, 1974; Paredes \& Tarazona, 1980), e idénticos con relación en la clase Mollusca, ya que son los mitílidos, también característicos para la zona intermedia del litoral en otros lugares del mundo (ej.: Esqueda et al. 2000; Araújo et al. 2005). La principal razón de su adaptación es que presentan mecanismos específicos de fijación como sus bisos que les permiten sujetarse a las rocas (Gosling, 2015), y muchas veces forman comunidades monoespecíficas dominada por una especie en particular (Paredes \& Tarazona, 1980). Para la franja infralitoral se obtuvo a T. atra como la especie más representativa, seguida de $P$. niger, composición similar encontrada en la costa del centro del Perú por Paredes (1974). Los tróchidos son comunes para la franja infralitoral, y debido a ello esta subzona es conocida también como la zona tróchido (Bhatt \& Bal, 1973; Jaiswar \& Kulkarni, 2001). En el caso de algunas partes de la costa oeste de América del Sur entre Perú y Chile, T. atra es una especie que está asociada a fondos rocosos en la zona intermareal y submareal, y es reconocida como uno de los herbívoros más abundantes y frecuentes sometidos constantemente a la depredación por parte de algunos equinodermos (Vasquez \& Buschmann, 1997). Aunque, las especies predominantes por cada subzona son las mismas para el centro de Perú y norte de Chile, este trabajo muestra que el patrón de zonación no cambia en una misma provincia biogeográfica marina.

La mayor riqueza de especies en la zona mediolitoral es debido a la gran cantidad de ambientes disponibles que permiten una mayor colonización de los organismos (Jiménez et al. 2004), característica que se ajusta a la hipótesis de la heterogeneidad ambiental (MacArthur, 1965; Levinton, 1982), donde explica que un mayor número de microambientes están relacionados directamente con la diversidad de un lugar. Vakily (1992), menciona que las conchas de los moluscos forman un sustrato seguro para la colonización de muchas especies, y dado que en la zona mediolitoral hay abundancia de mitílidos, esto ayuda a explicar claramente la mayor abundancia y riqueza de especies. 


\section{AGRADECIMIENTOS}

Un agradecimiento a Carlos Paredes, Yuri Hooker y Frank Cardoso por la ayuda brindada en la determinación de las especies de moluscos al igual que a Dulack Richards y Cristian Aldea por su ayuda y bibliografía brindada. A Rosaura González y Víctor Quipuscoa por sus sugerencias para este manuscrito y todos los ayudantes de campo, que sin ellos no hubiera sido posible llevar a cabo este estudio.

\section{BIBLIOGRAFÍA}

Ahmad, O., Fang, T. P. \& Yahya, K. (2011). Distribution of intertidal organisms in the shores of Teluk Aling, Pulau Pinang, Malaysia. Publ. Seto Mar. Biol. Lab., 41, 51-61. https://doi. org/10.5134/159483

Álamo, V. \& Valdivieso V. (1997). Lista sistemática de moluscos marinos del Perú. Callo, Perú: Multiformes, S. A.

Aldea, C. \& Valdovinos, C. (2005). Moluscos del intermareal rocoso del centro-sur de Chile $\left(36^{\circ}-38^{\circ}\right)$ : Taxonomía y clave de identificación. Gayana, 69(2), 364396. http://dx.doi.org/10.4067/S071765382005000200014

Aldea, C., Rosenfeld, S. \& Cárdenas, J. (2011). Caracterización de la diversidad de moluscos bentónicos sublitorales en Isla Carlos III y áreas adyacentes, Estrecho de Magallanes, Chile. An. Inst. Patagon., 39(2), 7389. https://doi.org/10.4067/S0718686X2011000200006

Araújo, R., Bárbara, I., Sousa-Pinto, I. \& Quintino, V. (2005). Spatial variabil- ity of intertidal rocky shore assemblages in the northwest coast of Portugal. Estuar. Coast. Shelf Sci., 64(1), 658-670. https://doi.org/10.1016/j. ecss.2005.03.020

Bandel, K. \& Wedler, E. (1987). Hydroid, amphineuran and gastropod zonation in the littoral of the Caribbean Sea, Colombia. Senckenb. Marit., 19(1-2), 1-129.

Bhatt, Y. M. \& Bal, D. V. (1973). Zonation of intertidal organisms on the rocky shores of Bombay. J. Mar. Biol. Assoc. India, 305-316.

Castro, P. \& Huber, M. E. (2003). Marine Biology. Nueva York, EE. UU.: McGraw-Hill.

Chelazzi, G. \& Vannini, M. (1980). Zonation of intertidal molluscs on rocky shores of Southern Somalia. Estuar. Coast. Shelf Sci., 10(1), 569-583. https://doi. org/10.1016/S0302-3524(80)80076-4

Chappuis, E., Terradas, M., Cefalì, M. E., Mariani, S. \& Ballesteros, E. (2014). Vertical zonation is the main distribution pattern of littoral assemblages on rocky shores at a regional scale. Estuar. Coast. Shelf Sci., 147, 113-122. https:// doi.org/10.1016/j.ecss.2014.05.031

Clarke, K. R. \& Warwick, R. M. (2001). Change in marine communities: an approach to statistical analysis and interpretation. Plymouth, United Kingdom: PRIMER-E Plymouth Laboratory.

Dall, W. H. (1910). Report on a Collection of Shells from Peru, with a Summary of the Littoral Marine Mollusca of the Peruvian Zoological Province. Proc. USNM., 37, 147-294.

Espoz, C. (2002). Ecología y evolución de patelogastrópodos endémicos a la costa rocosa de Perú y Chile: distribución, abundancia y filogenia. Tesis doctoral no publicada, Universidad Católica de Chile, Santiago, Chile. 
Espoz, C., Guzmán, G. \& Castilla, J. C. (1995). The lichen Thelidium litorale on shells of intertidal limpets: a case of lichen-mediated cryptic mimicry. Mar. Ecol. Prog. Ser., 119, 191-197. https:// doi.org/10.3354/meps119191

Espoz, C., Lindberg, D. R., Castilla, J. C. \& Simison, W. B. (2004). Los patelogastrópodos intermareales de Chile $\mathrm{y}$ Perú. Rev. Chil. Hist. Nat., 77(1), 257283. https://doi.org/10.4067/S0716078X2004000200006

Esqueda, M. del C., Ríos-Jara, E., Michel-Morfín, J. E. \& Landa-Jaime, V. (2000). The vertical distribution and abundance of gastropods and bivalves from rocky beaches of Cuastecomate Bay, Jalisco, México. Rev. Biol. Trop., 48(4), 765-775. PMid:11487925

Esqueda-González, M. C., Ríos-Jara, E., Galván-Villa, C. M. \& Rodríguez-Zaragoza, F. A. (2014). Species composition, richness, and distribution of marine bivalve molluscs in Bahía de Mazatlán, Mexico. ZooKeys, 399(1), 43-69. https://doi.org/10.3897/ zookeys.399.6256

Feinsinger P. (2004). El diseño de estudios de campo para la conservación de la biodiversidad. Santa Cruz de la Sierra, Bolivia: FAN.

Fernández, J. R. \& Jiménez, M. M. (2006). Estructura de la comunidad de moluscos y relaciones tróficas en el litoral rocoso del estado Sucre, Venezuela. Rev. Biol. Trop., 54(3), 121-130.

Galli, C. (2013). Worldwide mollusc species data base. Recuperado en setiembre 18, 2016, disponible en http://www.bagniliggia.it/WMSD/WMSDsearch.html.

Gosling, E. (2015). Marine Bivalve Molluscs. Chichester, United Kingdom: Wiley Backwell. https://doi. org/10.1002/9781119045212
Güller, M. \& Zelaya, D. G. (2013). The families Carditidae and Condylocardiidae in the Magellan and Peru-Chile provinces (Bivalvia: Carditoidea). Zootaxa, 3682(2), 201-239. https://doi. org/10.11646/zootaxa.3682.2.1

Guzmán, N., Saá, S. \& Ortlieb, L. (1998). Catálogo descriptivo de los moluscos litorales (Gastropoda y Pelecypoda) en la zona de Antofagasta, $23^{\circ} \mathrm{S}$ (Chile). Estud. Oceanol, 17(1), 17-86.

Hammer, Ø., Harper, D. A. T. \& Ryan, P. D. (2001). Paleontological statistics software package for education and data analysis. Palaeontol. Electron, 4(1), 9-18.

Hughe, R. A. (1991). Las aves de la provincia de Islay. Bol. Lima, 75, 47-54.

Jaiswar, A. K. \& Kulkarni, B. G. (2001). Vertical distribution of molluscs in the intertidal area in and around Mumbai, India. J. Indian Fish. Assoc., 28, 93-100.

Jiménez, M., Márquez, B. \& Díaz, O. (2004). Moluscos del litoral rocoso en cuatro localidades del Estado de Sucre, Venezuela. Saber, 16(1), 9-18.

Jones, W. E., (1980). Field teaching methods in shore ecology. In J. H. Price, D. E. G. Irvine \& W. F. Farnham (Eds.), The Shore Environment, Vol. I: Methods (pp. 19-44.) London, United Kingdom: Academic Press.

Krebs, C. J. (1985). Ecología, estudio de la distribución y la abundancia. México: Harla.

Levinton, J. S. (1982). Marine Ecology. Nueva Jersey, EE. UU.: Prentice-Hall.

Londoño-Cruz, E., López de Mesa-Agudelo, L. A., Arias-Galvez, F., Herrera-Paz, D. L., Prado, A., Cuellas, L. M. \& Cantera, J. (2014). Distribution of macroinvertebrates on intertidal rocky shores in Gorgona Island, Colombia (Tropical Eastern Pacific). Rev. Biol. Trop., 62(1), 
189-198. https://doi.org/10.15517/rbt. v62i0.16275

MacArthur, R. H. (1965). Patterns of species diversity. Biol. Rev., 40, 510533. https://doi.org/10.1111/j.1469185X.1965.tb00815.x

Marincovich L. J. (1973). Intertidal Mollusks of Iquique, Chile. Contr. Sci. Mus. Nat. Hist. Los Angeles, 16, 1-49.

McLean, J. H. (1984). Systematics of Fissurella in the Peruvian and Magellanic faunal provinces (Gastropoda: Prosobranchia). B. Nat. Hi. Mus. Angeles Country, 354(1), 1-76.

McQuaid, C. D. (1996). Biology of the gastropod family Littorinidae; I. Evolutionary aspects. Oceanogr. Mar. Biol. Ann. Rev. 34, 233-262.

Moore, B. H. (1958). Marine Ecology. Nueva York, EE. UU.: Wiley \& Sons.

Ojeda, J., Rosenfeld, S., Marambio, J., Rozzi, R. \& Mansilla, A. (2014). Patrones estacionales y espaciales de la diversidad de moluscos intermareales de bahía Róbalo, canal Beagle, Reserva de la Biosfera Cabo de Hornos, Chile. Rev. Biol. Mar. Oceanogr., 49, 493509. https://doi.org/10.4067/S071819572014000300007

Osorio, C. \& Cantuarias, V. (1989). Vertical distribution of mollusks on the rocky intertidal of Easter Island. Pac. Sci., 43(4), 302-315.

Paredes, C. (1974). El modelo de zonación en la orilla rocosa del departamento de Lima. Rev. Peru. Biol., 1(2), 168191. http://dx.doi.org/10.15381/rpb. v1i2.8422

Paredes, C. (1980). La familia Acmaeidae (Gastropoda, Archaeogastropoda) en el Perú. Rev. Peru. Biol., 2(1), 5258. http://dx.doi.org/10.15381/rpb. v2i1.8359

Paredes, C. (1986). La familia Fissurellidae (Gastropoda, Archaeogastropoda) en el Perú. Rev. Cienc. UNMSM, 74(1), 75-86.

Paredes, C., Cardoso, F., Santamaría, J., Esplana, J. \& Llaja, J. (2016). Lista anotada de los bivalvos marinos del Perú. Rev. Peru. Biol., 23(2), 127-150. https://doi. org/10.15381/rpb.v23i2.12397

Paredes, C., Cardoso, F. \& Tarazona, J. (1999). Invertebrados del intermareal rocoso del departamento de Lima, Perú: una lista comentada de especies. Rev. Peru. Biol., 6(1), 5-47. http://dx.doi. org/10.15381/rpb.v6i2.8309

Paredes, C. \& Tarazona, J. (1980). Las comunidades de mítilidos del mediolitoral rocoso del departamento de Lima. Rev. Peru. Biol., 2(1), 59-72. http://dx.doi. org/10.15381/rpb.v2i1.8362

Ramírez, R., Paredes, C. \& Arenas, J. (2003). Moluscos del Perú. Rev. Peru. Biol., 51(3), 225-284. https://doi. org/10.15517/rbt.v51i3.26386

Schrödl, M. \& Hooker, Y. (2014). Sea slugs of Peru: Peruvian-Chilean faunal elements. Spixiana, 37(1), 45-59.

Shick, J. M., Widdows, J. \& Gnaiger, E. (1988). Calorimetric studies of behavior, metabolism and energetics of sessile intertidal animals. Am. Zool., 28(1), 161-181. https://doi.org/10.1093/ $\mathrm{icb} / 28.1 .161$

Sibaja-Cordero, J. A. (2008). Vertical zonation in the rocky intertidal at Cocos Island (Isla del Coco), Costa Rica: A comparison with other tropical locations. Rev. Biol. Trop., 56(2), 171-187. https://doi. org/10.15517/rbt.v56i2.27017

Sibaja-Cordero, J. A. \& Cortés, J. (2008). Vertical zonation of rocky intertidal organisms in a seasonal upwelling area (Eastern Tropical Pacific), Costa Rica. Rev. Biol. Trop., 56(4), 91-104. https:// doi.org/10.15517/rbt.v56i4.27208

Sokal, R. R. \& Rohlf, F. J. (1981). Biometry. The principles and practice of statistics 
in biological research. Nueva York, EE. UU.: W. H. Freeman \& Company. Spalding, M. D., Fox, H. E., Allen, G. R., Davidson, N., Ferdaña, Z. A., Finlayson, M., Robertson, J. (2007). Marine ecoregions of the world: A bioregionalization of coastal and shelf areas. BioScience, 57(7), 573-583. https://doi. org/10.1641/B570707

Stephenson, T. A., \& Stephenson, A. (1949). The universal features of zonation between tide-marks on rocky coasts. J. Ecol., 37(2), 289-305. https://doi. org/10.2307/2256610

Talledo, C. R. \& Gonzales., R. (1989). Moluscos Gastrópodos y Placóforos de la Provincia de Islay (Arequipa), Perú. Arequipa, Perú: Universidad Nacional de San Agustín.

Torreblanca-Ramírez, C., Flores-Garza, R., Flores-Rodríguez, P., García-Ibañez, S. \& Galeana-Rebolledo, L. (2012). Riqueza, composición y diversidad de la comunidad de moluscos asociada al sustrato rocoso intermareal de playa Parque de la Reina, Acapulco, México. Rev. Biol. Mar. Oceanogr., 47(2), 283294. https://doi.org/10.4067/S071819572012000200010

Underwood, A. J. (1981). Structure of a rocky intertidal community in New South Wales: Patterns of vertical distribution and seasonal changes. J. Exp. Mar. Biol. Ecol., 51(1), 57-85. https://doi. org/10.1016/0022-0981(81)90154-4
Vakily, J. M. (1992). Determination and Comparison of Bivalve Growth, with Emphasis on Thailand and Other Tropical Areas. Manila, Philippines: ICLARM Technical Reports 36.

Vasquez, J. A. \& Buschmann, A. H. (1997). Herbivore-kelp interactions in Chilean subtidal communities: a review. Rev. Chil. Hist. Nat., 70, 41-52.

Vegas-Vélez, M. (1971). Introducción a la ecología de bentos marino. Programa regional de desarrollo científico y tecnológico. Serie de Biología 9. Washington, D. C., EE. UU.: OEA.

Veliz, D. \& Vásquez, J. A. (2000). La familia Trochidae (Mollusca: Gastropoda) en el norte de Chile: consideraciones ecológicas y taxonómicas. Rev. Chil. Hist. Nat., 73(1), 757-769. https://doi.org/10.4067/S0716078X2000000400018

Veliz, D., Winkler, F. M., Guisado, C. \& Collin, R. (2012). A new species of Crepipatella (Gastropoda: Calyptraeidae) from northern Chile. Molluscan Res., 32(3), 145-153.

Wieser, W. (1980). Metabolic and products in three species of marine gastropods. J. Mar. Biolog. Assoc. U.K., 60(1), 175-180. https://doi.org/10.1017/ S0025315400024231

WoRMS Editorial Board. (2017). World register of marine species. Recuperad en octubre 10, 2017, disponible en http://www.marinespecies.org. doi: $10.14284 / 170$ 